EPJ Web of Conferences 60, 20029 (2013)

DOI: $10.1051 /$ epjconf $/ 20136020029$

(C) Owned by the authors, published by EDP Sciences, 2013

\title{
Search for a Higgs-like boson decaying into bottom quarks in the Z(II)H chan- nel
}

\author{
Philipp Eller ${ }^{1, a}$, on behalf of the CMS collaboration \\ ${ }^{1}$ Institute for Particle Physics, ETH Zurich
}

\begin{abstract}
After the discovery of a Higgs-like Boson with a mass close to $125 \mathrm{GeV}$ at the LHC in summer 2012, we are showing the update on the analysis of the VH cannel. In this channel the Higgs-like Boson is produced in association with a vector boson and decaying into b quarks. We present the updated results on the full 2011 and $20127+8 \mathrm{TeV}$ dataset. This poster will focus on one of the tree modes that are combined in this analysis, where the associated vector boson is a $\mathrm{Z}$ boson that is decaying leptonically into two electrons or muons, respectively.
\end{abstract}

\section{Introduction}

We perform a search for the standard model decay $\mathrm{H}$ to bb with the CMS detector at the LHC [1] in events where the Higgs boson is produced in association with a vector boson. The analysis presented here contains approximately $7 \mathrm{fb}^{-1}$ more $8 \mathrm{TeV}$ data and some modifications with respect to the previous CMS Higgs boson search in these final states [2].

\section{Analysis}

\subsection{Analysis strategy}

In the associated production topology the analysis is devided into three modes. This analysis is studying associated production with a $\mathrm{Z}$ boson decaying leptonically, see Figure 1. One of the other two modes contain a $\mathrm{Z}$ boson decaying invisibly and in the third mode the Higgs is produced in association with a $\mathrm{W}$ which then decays semileptonically. The presence of a vector boson in the final state highly suppresses the QCD background, while requiring a large boost reduces the large backgrounds from $\mathrm{W}$ and $\mathrm{Z}$ production in association with jets. The boost also generally improves the mass resolution of the reconstructed Higgs candidates.

The dominant backgrounds encountered in this analysis arise from three general sources:

- production of $\mathrm{Z}$ bosons in association with one or more jets;

- production of ttbar pairs, as well as single top quarks;

- production of vector-boson pairs.

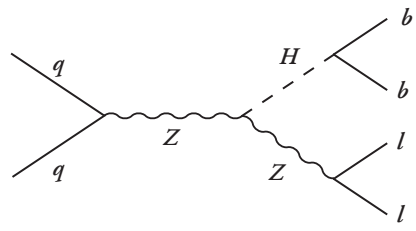

Figure 1. Tree-level Feynman diagram of the associated productionm of Higgs boson decaying into two b-quarks with a Z-boson decaying leptonically.

\subsection{Signal and control regions}

In this topology we are mostly interested in a region requiring a large boost, thus we categorize in Vector $\mathrm{p}_{\mathrm{T}}$ and have a high sensitivity bin for vector-boson $\mathrm{p}_{\mathrm{T}}(\mathrm{V})>$ $100 \mathrm{GeV}$ and a less sensitive bin with $\mathrm{p}_{\mathrm{T}}(\mathrm{V})$ between 50 and $100 \mathrm{GeV}$. This categorization is done for the electron and muon cases, resulting in four bins that are combined in a single fit. A detailed overview of the signal regions is given in Table 2. Control regions enriched in $\mathrm{Z}+$ light jets, $\mathrm{Z}+\mathrm{b}$-jets, and tt-bar events are used to assure Data/MC agreement for our BDT input variables and to verify the BDT classifiers. Also the normalization of tt-bar, DY+light jets, DY+1 b-jet and DY +2 b-jets are fitted simultaneously in control regions that are very close to the signal region, together for electrons and muons, considering the electron and muon efficiencies as additional nuisances. Scale factors are summarized in Table 1.

Table 1. Scale factors for the Drell-Yan and ttbar simulated samples derived from a simulatneous fit in mass sidebands.

\begin{tabular}{ll} 
DY +0 b-jets & $1.11 \pm 0.04 \pm 0.06$ \\
$D Y+1$ b-jet & $1.59 \pm 0.26 \pm 0.08$ \\
DY +2 b-jets & $0.98 \pm 0.10 \pm 0.08$ \\
tt-bar & $1.10 \pm 0.05 \pm 0.06$ \\
\hline
\end{tabular}

\footnotetext{
a e-mail: peller@phys.ethz.ch
} 
Table 2. Definition of the signal regions for the BDT and the Mjj analyses

\begin{tabular}{lcccccccc} 
Variable & $\mathrm{p}_{\mathrm{T}}\left(\mathrm{j}_{1}\right), \mathrm{p}_{\mathrm{T}}\left(\mathrm{j}_{2}\right)$ & $m(\mathrm{Z})$ & $m(\mathrm{H})$ & $\mathrm{p}_{\mathrm{T}}(\mathrm{Z})$ & $\mathrm{CSV}_{\max }$ & $\mathrm{CSV}_{\min }$ & $\mathrm{MET}$ & $\Delta \mathrm{R}(\mathrm{jj})$ \\
\hline $\begin{array}{l}\text { BDT region } \\
\text { high } \mathrm{p}_{\mathrm{T}}(\mathrm{V})\end{array}$ & $>20 \mathrm{GeV}$ & {$[75-105] \mathrm{GeV}$} & $<250 \mathrm{GeV}$ & $>100 \mathrm{GeV}$ & $>0.244$ & $>0.244$ & - & - \\
low $\mathrm{p}_{\mathrm{T}}(\mathrm{V})$ & $>20 \mathrm{GeV}$ & {$[75-105] \mathrm{GeV}$} & {$[40-250] \mathrm{GeV}$} & {$[50,100] \mathrm{GeV}$} & $>0.5$ & $>0.244$ & - & - \\
\hline $\begin{array}{l}\text { Mjj region } \\
\text { high } \mathrm{p}_{\mathrm{T}}(\mathrm{V})\end{array}$ & $>20 \mathrm{GeV}$ & {$[75-105] \mathrm{GeV}$} & - & $>150 \mathrm{GeV}$ & $>0.679$ & $>0.5$ & $<60 \mathrm{GeV}$ & $<1.6$ \\
medium $\mathrm{p}_{\mathrm{T}}(\mathrm{V})$ & $>20 \mathrm{GeV}$ & {$[75-105] \mathrm{GeV}$} & - & {$[100,150] \mathrm{GeV}$} & $>0.679$ & $>0.5$ & $<60 \mathrm{GeV}$ & - \\
low $\mathrm{p}_{\mathrm{T}}(\mathrm{V})$ & $>20 \mathrm{GeV}$ & {$[75-105] \mathrm{GeV}$} & - & {$[50,100] \mathrm{GeV}$} & $>0.679$ & $>0.5$ & $<60 \mathrm{GeV}$ & - \\
\hline
\end{tabular}

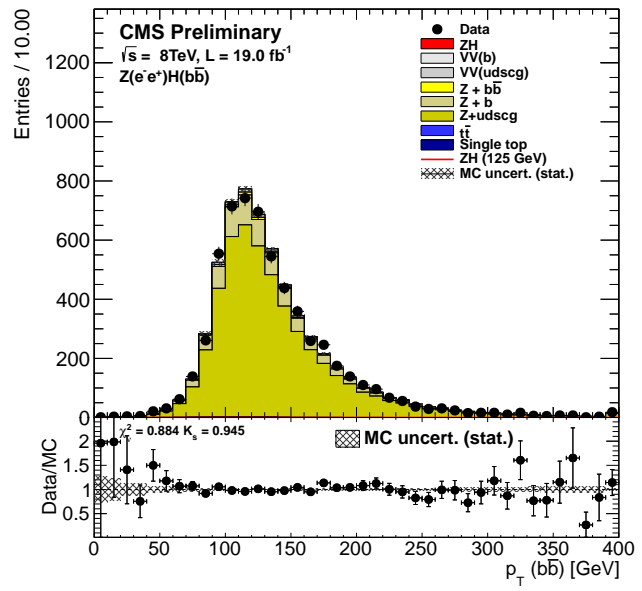

Figure 2. Distribution of the dijet pt for simulated samples in the $\mathrm{Z}+$ light jets control region for the electron channel.

\subsection{Regression}

The resolution on the dijet mass is improved significantly using a regression technique with a BDT. A correction is computed for individual jets that attempts to more accurately estimate the true b-jet energy, thus improving the resolution and reducing the scale bias on a per-jet basis. As input variables we use different Jet properties like vertex information, jet energy scale uncertainty, soft lepton properties, particle-flow missing transverse energy and others. The resulting improvement in the dijet mass resolution is approximately $20 \%$ in this channel, Figure 3 . This regression technique is validated on data in the different control regions. Figure 4 shows the ratio between the dijet $\mathrm{p}_{\mathrm{T}}$ and the $\mathrm{p}_{\mathrm{T}}$ of the dilepton system before and after regression, respectively.

\subsection{Cross-checks}

In addition to the main MVA based analysis we carry out a simpler analysis on the dijet mass spectrum. This serves as a cross-check of our main analysis and also for visualization purposes. The sensitivity is around $40 \%$ lower than with the BDT approach, which mainly takes advantage of the correlations of the input variables. A combined plot of the $\mathrm{M}_{\mathrm{jj}}$ spectrum in the signal regions is shown in Figure 5 .

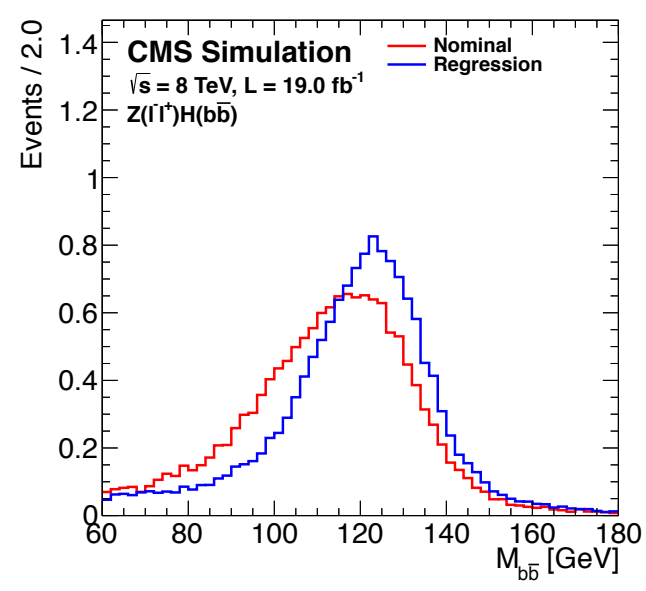

Figure 3. Distributions of dijet invariant mass in signal $\mathrm{Z}(11) \mathrm{H}$ events before and after the regression is applied.
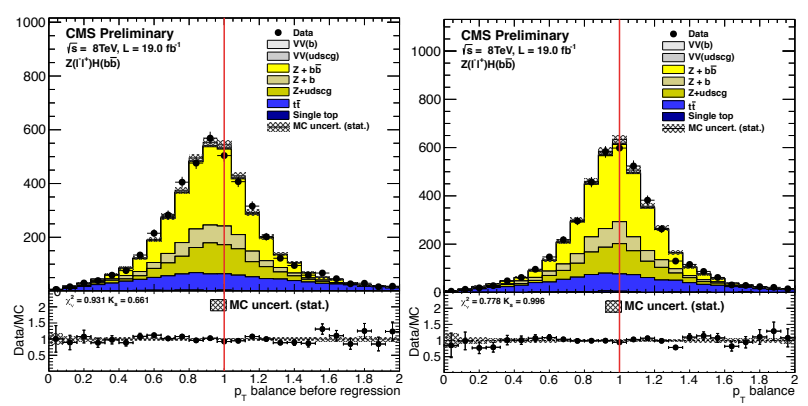

Figure 4. Distribution of the ratio between the dijet $\mathrm{p}_{\mathrm{T}}$ and the $\mathrm{p}_{\mathrm{T}}$ of the dilepton system on data versus MC before (left) and after (right) regression. Data to Monte Carlo agreement is evidently assured.

\section{Results}

One of the four BDT distributions is given as an example in Figure 6, on which the limits are derived and signals extracted. Taking into account all systematic uncertainties and a combination with the other analysis channels, $\mathrm{Z}(\mathrm{nn}) \mathrm{H}$ and $\mathrm{W}(\mathrm{ln}) \mathrm{H}$, plus $7 \mathrm{TeV}$ data gives an expected upper Limit of 0.95 times the standard model expectation whereas we observe 1.89 times the standard model expectation for a mass hypothesis of $125 \mathrm{GeV}$. Individual Limits for several mass hypotheses at $8 \mathrm{TeV}$ for this analysis channel are shown in Figure 7. The local expected signifi- 


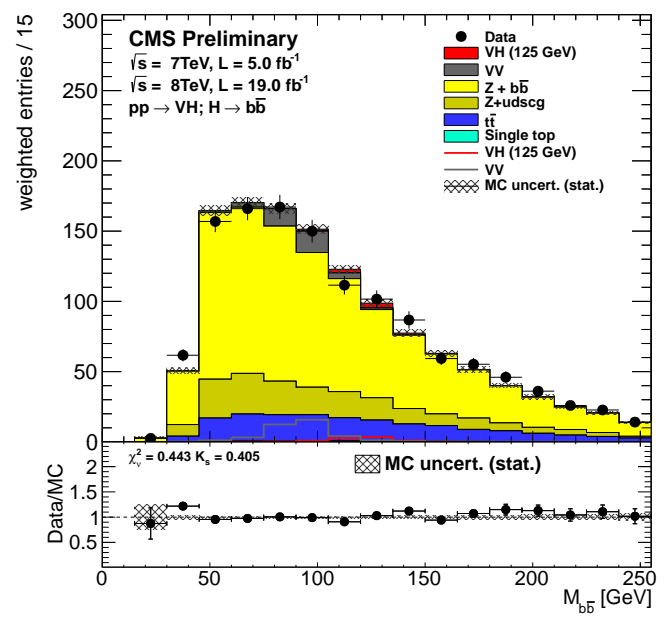

Figure 5. This combined $\mathrm{M}(\mathrm{jj})$ plot of all our $\mathrm{Z}(11) \mathrm{H}$ analysis bins is weighted by $\mathrm{s} /(\mathrm{s}+\mathrm{b})$ (in a window of $\pm 20 \mathrm{GeV}$ around $125 \mathrm{GeV}$ ) for both 7 and $8 \mathrm{TeV}$ datasets. In this plot the post-fit normalization factors have been applied.

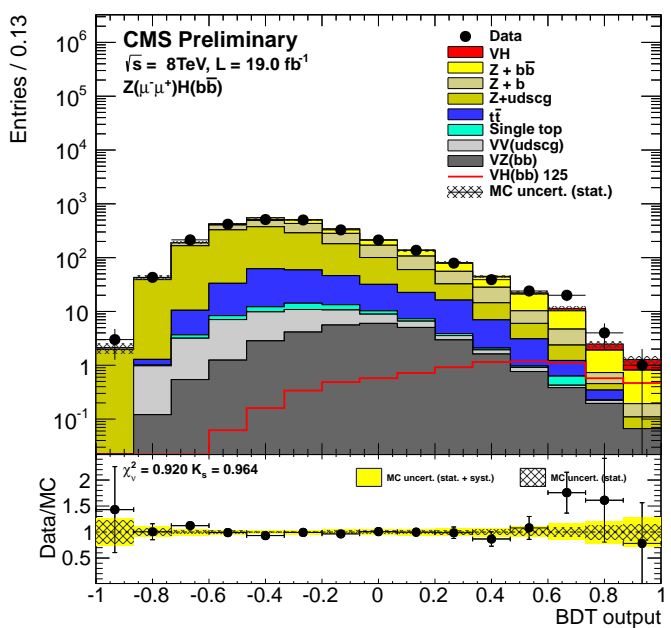

Figure 6. Distribution of the BDT output for simulated samples in the hight $\mathrm{p}_{\mathrm{T}}(\mathrm{V})$ muon signal region. It show overall very good agreement with the data.

cance for a SM Higgs boson of mass $m_{H}=125 \mathrm{GeV}$ is 2.1 and we observe 2.1 in the combination with the other two channels [3]. In this channel alone, the best fit of the signal strength is $0.82 \pm 0.97$ and agrees very well with the standard model prediction and is compatible with the two other channels of our analysis, c.f. Figure 8. In the combination we get $1.0 \pm 0.5$ times the SM cross section with the main BDT analysis and with the $\mathrm{M}(\mathrm{jj})$ cross-check Analysis 0.8 $\pm 0.7[3]$.

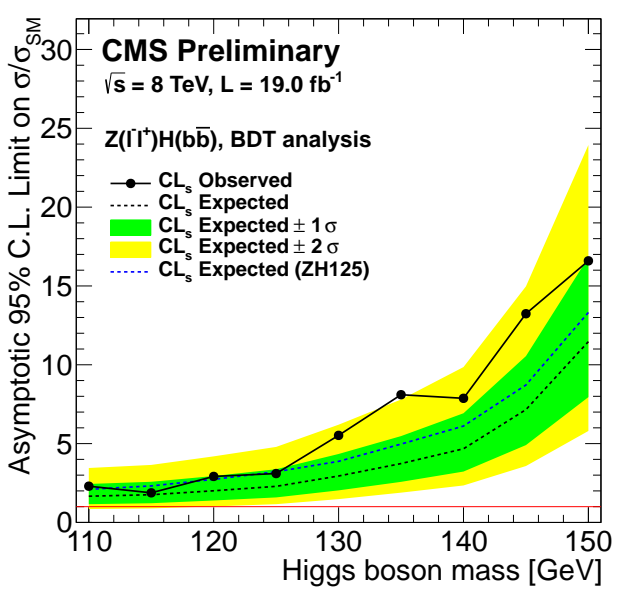

Figure 7. Expected and observed 95\% CL upper limits on the product of the $\mathrm{VH}$ production cross section times the $\mathrm{Hbb}$ branching ratio, with respect to the expectations for a standard model Higgs boson. The blue dashed line represents the expected observed limits obtained from replacing the data with the sum of expected background and signal for a Higgs boson.

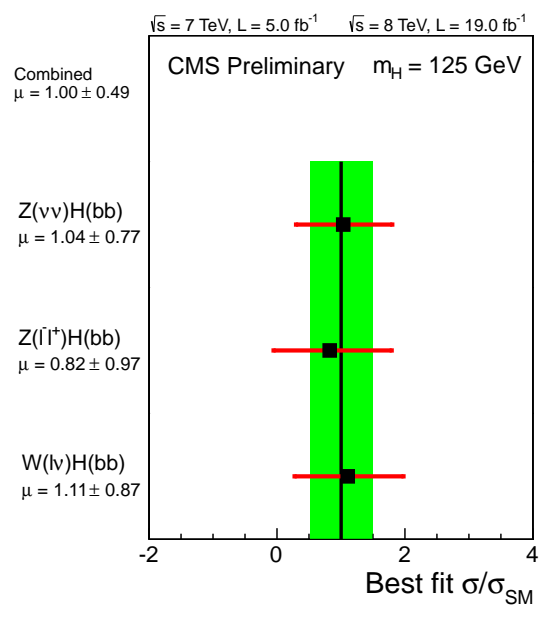

Figure 8. The most likely value of the production cross section for a $125 \mathrm{GeV}$ Higgs boson, relative to the standard model cross section, for each channel and for all channels combined (green band).

\section{References}

[1] CMS Collaboration, Journal of Instrumentation JINST 3 S08004 (2008), "The CMS experiment at the CERN LHC".

[2] CMS Collaboration, CMS Physics Analysis Summary CMS-PAS-HIG-12-044 (2012), "Search for the standard model Higgs boson produced in association with $\mathrm{W}$ or $\mathrm{Z}$ bosons, and decaying to bottom quarks for HCP 2012".

[3] CMS Collaboration, CMS Physics Analysis Summary CMS-PAS-HIG-13-012 (2013), "Search for the standard model Higgs boson produced in association with $\mathrm{W}$ or $\mathrm{Z}$ bosons, and decaying to bottom quarks (LHCP 2013)". 